\title{
OPTIMIZATION OF THE PERFORMANCE OF MULTI-ARM SURGICAL ROBOT AND CONSTRUCTION OF VIRTUAL SIMULATION SYSTEM
}

\author{
Qian Li $^{1}$, Meiyu Chen ${ }^{1}$, Zijian Gao ${ }^{1}$ \\ ${ }^{1}$ Electric Information College of Changchun Guanghua University, Changchun, 130031, China. \\ Email: liqianliqiandrme2@yeah.net
}

\begin{abstract}
To optimize the performance of the multi-arm surgical robot and apply it in the virtual simulation system, so as to achieve better surgical results, in this research, by analyzing the joint and position characteristics of the robotic arm for multi-arm surgical robot, the variables that need to be optimized are determined, and the operating space is divided. According to the performance index of the multi-arm surgical robot, the global isotropic function, multi-arm cooperation function, and multi-arm maximum distance function are constructed, and a comprehensive optimization algorithm combining particle swarm optimization algorithm with gaussian process is proposed. Based on this, the virtual simulation system is constructed, the weight distribution of the subspace is set, and the iterative experiment is carried out in the virtual simulation system to compare the optimization speed and accuracy of the proposed comprehensive optimization algorithm with the particle swarm optimization algorithm, so as to verify the effectiveness and rationality of the proposed algorithm. The results show that with the increase of iteration times, the values of global isotropic optimization function and the multi-arm cooperation function increase gradually, the efficiency of the multi-arm surgical robot also increases, and the proposed algorithm is effective and feasible. And the higher the set weight, the larger the simulated cooperative surgical space, which can be used as a surgical hotspot for the robotic arm positioning. The total value of the integrated objective optimization function obtained by the proposed algorithm is larger than that of the particle swarm optimization algorithm, with higher precision and fewer iterations. Without considering the weight of the subspace, it is impossible to guarantee that the multi-arm surgical robot has a high efficiency in the hot spot area. Therefore, the effectiveness of the multi-arm surgical robot can be optimized according to the algorithm proposed in this study, so as to achieve a good surgical effect and provide guidance for clinical use in the future.
\end{abstract}

Keywords: Multi-Arm Surgical Robot; Performance Optimization Function; Comprehensive Optimization Algorithm; Particle Swarm Optimization Algorithm; Virtual Simulation System.

\section{Introduction}

With the rapid development of minimally invasive surgery, doctors no longer need to touch the patient when performing the surgery, but perform the operation with instruments and equipment. Compared with traditional medical surgery, minimally invasive surgery has smaller wounds and allows patients to recover faster [1].However, minimally invasive surgery has some defects, such as lack of three-dimensional field of vision and wrist movement, and its application is limited to some extent [2].Compared with traditional minimally invasive surgery, robot-assisted surgery can solve these problems. Doctors can operate a robot to perform surgery without the effects of arm tremors caused by fatigue [3].Surgical robot can replace human for surgical operation, expanding human's ability to coordinate operation. The combination of minimally invasive surgery and surgical robot has important practical value.
During minimally invasive surgery, oblique devices need to be inserted into the body of the patient, so doctors need to determine the location of the surgical incision and the preoperative location of the robotic arm to achieve the best surgical effect [4]. However, due to the complex structure of the multiarm surgical robot, which requires the coordination of multiple armed arms, doctors can only perform preoperative positioning of the surgical robot based on surgical experience, which can't guarantee the realization of the maximum efficiency of the multiarm surgical robot [5]. Therefore, it is very important to establish the efficiency optimization model algorithm of multi-arm surgical robot. At the same time, the patient's life safety should also be guaranteed during the operation, and preoperative planning and rehearsal are usually carried out on animal carcasses, which is expensive and not in line with ethical requirements [6]. On the contrary, virtual simulation system based on multi-arm 
surgical robot can reduce the cost and time, and the rehearsal environment is safe and real.

The virtual simulation system can set the surgical level according to the difficulty of surgery and the level of doctors, which can not only enable young doctors to master surgical skills, but also enable experienced doctors to maintain the level of surgery [7].

Based on this, in this research, by analyzing the joint and position characteristics of the robotic arm of a multi-arm surgical robot, the variables to be optimized are determined. According to the performance index of the multi-arm surgical robot, the performance optimization function is constructed, and a comprehensive optimization algorithm combining particle swarm optimization algorithm with gaussian process is proposed. A virtual simulation system is constructed and iterative experiments are carried out in the virtual simulation system to verify the effectiveness and rationality of the proposed comprehensive optimization algorithm. It is expected to improve the performance of multi-arm surgical robot and provide guidance for clinical application in the future.

\section{Literature Review 2.1 Foreign research progress}

Aldair et al. (2019) proposed an adaptive fuzzy controller (AFC) based on ant colony optimization (ACO) to eliminate the vibration phenomenon of surgical robots moving on rough surfaces.

The results show that the stability of the adaptive fuzzy controller is relatively high [8]. Ros-Freixedes et al. (2019) proposed an optimization framework to find the optimal design parameters for the surgical robot with unsteady curvature to reach the remote target, and try to avoid collision with the surrounding tissues at the same time.

The results show that this method is highly feasible, and the cost function value of the optimal design of unsteady curvature is lower than that of the standard constant curvature robot [9]. Watanabe et al. (2017) proposed a system consisting of single host and double slave units to make the operation more efficient, and found that the operation time was reduced by $20 \%$ in total [10].

\subsection{Domestic research progress}

Zhang et al. (2019) introduced a new type of dual-arm coordination impedance into multi-priority control for redundant dual-arm robots, and improved the performance of target tracking and redundant joints by adjusting the relative cartesian error between the two arms.

The results showed that the proposed dual-arm coordination impedance can directly adjust the relative tracking error between the two objects, and can successfully achieve the dual-arm task of nailing holes under the external impact force applied to the two end-effectors [11].Wang et al. (2018) proposed an automatic tracking algorithm based on the mixed gray prediction model, which was applied to the laparoscopic visual window of the automatic navigation robot-assisted surgery system, and simulated the real motion trajectory of the main manipulator.

The results showed that the algorithm had high accuracy, practical feasibility, and robustness [12].Zhang et al. (2017) proposed a new practical method to optimize the parameters of surgical robot terminal tools, so as to complete hand-eye calibration.

The results showed that the one-time rectification method can significantly improve the efficiency of hand-eye calibration of robot, optimize the absolute positioning accuracy of the one-time registration method, and meet the requirements of clinical surgery [13].

Through the related research at home and abroad, it is found that the research on surgical robots has made great progress, which has improved the efficiency of surgical robots to some extent. However, there are still few studies on the cooperation efficiency and preoperative positioning between robotic arms, and most of them have not been verified in the virtual simulation system. Based on this, in this research, the joint and position characteristics of the robotic arm of the multi-arm surgical robot are analysed. According to the performance index of the multi-arm surgical robot, the performance optimization function is constructed.

A particle swarm optimization algorithm combined with gaussian process is proposed to construct a virtual simulation system.

An iterative experiment is carried out in a virtual simulation system to verify the effectiveness and rationality of the proposed comprehensive optimization algorithm.

\section{Methods}

\subsection{Analysis of preoperative positioning of multi-arm surgical robot}

Most of the multi-arm surgical robots have three robotic arms, the middle of which is the mirror arm, and the end of which is equipped with a laparoscope.

The left and right arms are the holding arms, the ends of which are equipped with micro instruments.

As shown in Figure 1, the robotic arm is mounted on the base through joint 1 . Each robotic arm contains four passive joints of joints $1,2,3$, and 4 .

The relevant electromagnetic clutch settings are adjusted preoperatively, but don't change during the operation. 
The four active joints, such as joint $5,6,7$, and 8 , are operated and controlled by the doctor during the operation.

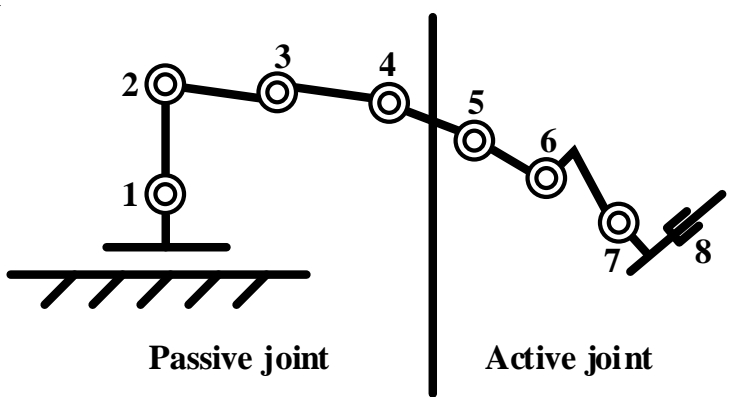

Figure 1: Structure of a multi-arm surgical robot
To analyse the kinematic coordinates of the surgical robot, an active joint kinematic coordinate diagram is established, as shown in Figure 2. The position coordinates of joint 4 are set as $P\left(p_{x}, p_{y}, p_{z}\right)$, and the rotation angle is $\theta$. Given the values of $P$ and $\theta$, the unique position coordinates of the passive joint can be obtained.

When the robot makes telecentric motion, the position of the active joint keeps changing, while the position of the telecentric point remains unchanged.

Therefore, by determining a set of values of $P$ and $\theta$, the coordinates of the position of the telecentric point can be determined.

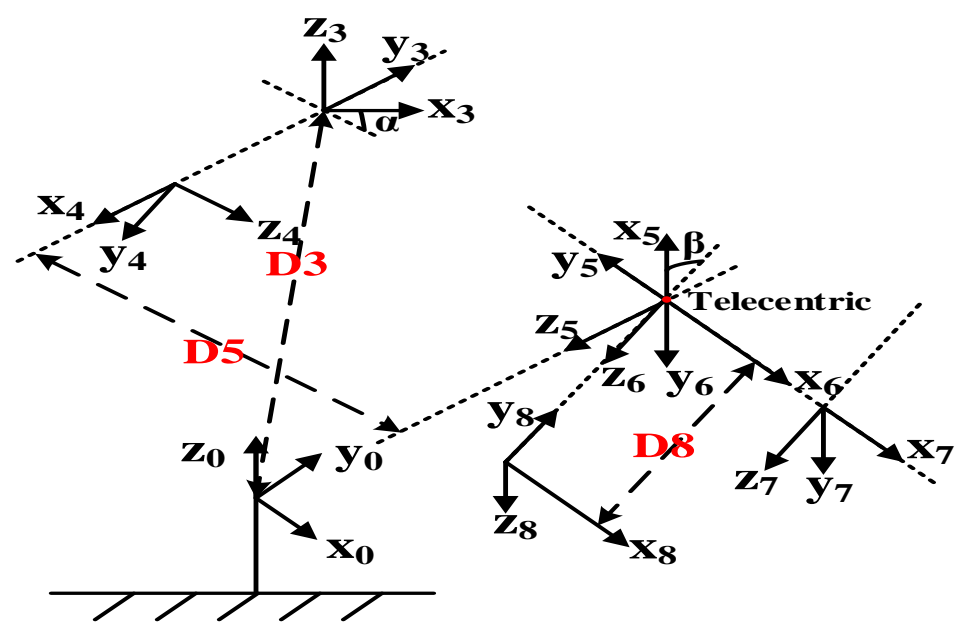

Figure 2: Kinematic coordinates of the active joint of a multi-arm surgical robot

According to the kinematic coordinate diagram of the active joint shown in Figure 2, the angle of joint 5 is $\theta_{5}$, which represents the overall rotation of the active joint of the robotic arm. The rotation angle of the joint 6 is $\theta_{6}$, which represents the abduction angle of the parallelogram structure. $\alpha$ and $\beta$ represent the structural parameters of the mechanical arm, so as to obtain the DH parameters of the active joint of the mechanical arm shown in Table 1.

Table 1. DH parameters of the active joint of the mechanical arm of the multi-arm surgical robot

\begin{tabular}{|c|c|c|c|c|c|c|}
\hline Joint & 3 & 4 & 5 & 6 & 7 & 8 \\
\hline$L_{i} / \mathrm{mm}$ & 0 & 0 & 90 & 0 & 90 & 120 \\
\hline$\alpha_{i} /^{\circ}$ & 0 & {$[-90-\alpha,-90]$} & -90 & {$[-90-\beta,-90]$} & 0 & -90 \\
\hline$D_{i} / \mathrm{mm}$ & $\mathrm{D} 3$ & 0 & D5 & 0 & 0 & D8 \\
\hline$\theta_{i} /^{\circ}$ & 0 & $\theta_{4}$ & $\theta_{5}$ & $\theta_{6}$ & $\theta_{7}$ & 0 \\
\hline
\end{tabular}

According to DH parameters, the following transformation matrix can be obtained.

$$
\begin{aligned}
{ }^{0} F_{8} & ={ }^{0} F_{3}{ }^{3} F_{4}{ }^{4} F_{5}{ }^{5} F_{6}{ }^{6} F_{7}{ }^{7} F_{8} \\
{ }^{0} F_{3} & =\operatorname{Trans}\left(x_{0}, p_{x}\right) \operatorname{Trans}\left(y_{0}, p_{y}\right) \operatorname{Trans}\left(z_{0}, p_{z}\right) \\
{ }^{3} F_{4} & =\operatorname{Rot}\left(z_{3}, \theta_{4}\right) \operatorname{Rot}\left(x_{4},\left(-\pi / 2-p_{1}\right)\right) \\
{ }^{4} F_{5} & =\operatorname{Rot}\left(z_{4}, \theta_{5}\right) \operatorname{Trans}\left(z_{4}, D_{5}\right) \operatorname{Rot}\left(x_{5},-\pi / 2\right) \\
{ }^{5} F_{6} & =\operatorname{Rot}\left(z_{5}, \theta_{6}\right) \operatorname{Rot}\left(x_{6}, \pi / 2\right) \\
{ }^{6} F_{7} & =\operatorname{Rot}\left(z_{6}, \theta_{7}\right) \\
{ }^{7} F_{8} & =\operatorname{Trans}\left(z_{7}, D_{8}\right) \operatorname{Rot}\left(z_{8},-\pi / 2\right)
\end{aligned}
$$


According to Equation 1, if the parameters $\theta_{5}$ and $\theta_{6}$, as well as the parameters $P$ and $\theta$ of joint 4 , can be determined, the preoperative position of the robotic arm of the surgical robot can be determined. The active joint is controlled by the surgeon intra operatively, and the parameters $\theta_{5}$ and $\theta_{6}$ affect the effectiveness of the surgical robot.

The effectiveness of a multi-arm surgical robot is closely related to its performance in the workspace, and a robot efficiency optimization model can be constructed in the workspace. The internal differences in the operating space are reflected in the whole process of robot-assisted surgery, and the positions of the arm holding the laparoscope and the mechanical arm are constantly adjusted to achieve the surgical effect. To reflect the internal differences of the surgical workspace, the surgical workspace is divided into the structure shown in Figure 3.



Figure 3: Surgery workspace of multi-arm surgical robot

The surgical workspace is simplified into a hexahedral structure, and the subdivision mainly includes the number of subspaces and the volume of subspaces.

Along the $x$-axis, $y$-axis, and z-axis, the workspace is divided into $a, b$, and $c$ parts respectively.

Each part can be represented as $a_{i}(i=1,2,3, \ldots, \mathrm{A}), b_{i}$ $(i=1,2,3, \ldots, \mathrm{B})$, and $c_{i}(i=1,2, \ldots, \mathrm{C})$, each subspace can be represented as $S_{i j k \text {. }}$

According to surgical requirements, the volume of each subspace is approximately $(7-10) \mathrm{cm} \times(7-10)$ $\mathrm{cm} \times(7-10) \mathrm{cm}$.

Due to the limitation of human body, the maximum values of $A, B$, and $C$ are set as 3,3 , and 2 respectively.

\subsection{Construction of performance optimization function of multi-arm surgical robot}

First, the optimization function of single-arm isotropic performance

Single-arm isotropy refers to that the robot can move in any direction under the specified position. The Jacobian matrix of the robot arm is constructed by the vector product method, as shown in the following equation.

$$
J_{i}=\left[\begin{array}{c}
J_{l i} \\
J_{a i}
\end{array}\right]=\left[\begin{array}{c}
b_{i-1} \\
0
\end{array}\right]
$$

$$
J_{i}=\left[\begin{array}{c}
J_{l i} \\
J_{a i}
\end{array}\right]=\left[\begin{array}{c}
b_{i-1} \times r_{i-1, e} \\
b_{i-1}
\end{array}\right]
$$

Where, $J_{l i}$ and $J_{a i}$ respectively represent the threedimensional linear velocity and angular velocity coefficients generated by the joint $i$ variable. $b_{i-1}$ represents the unit vector in the direction of the $z_{i-1}$ axis, $r_{i-1, e}$ represents the vector from the origin of the $o_{i-1}$ coordinate system to the terminal coordinate system $o_{n}$.

For the multi-arm surgical robot, the Jacobian matrix of active and passive joint 4 of the arm holding the robot is established as follows:

$$
J=\left(J_{4}, J_{5}, J_{6}, J_{7}\right)
$$

However, adopting only the Jacobian matrix has certain defects, which can only reflect the local characteristics of the robot. On this basis, the global isotropic optimization function (GII) is constructed by combining the partition of workspace and weight distribution, as shown in Equation 5.The larger the GII, the more the single robot arm of the surgical robot tends to be isotropic.

$$
G I I=\sum_{i=0}^{A} \sum_{j=0}^{B} \sum_{k=0}^{C} \omega_{i j k} \gamma_{i j k} \frac{\min _{0} \in S_{i j k}}{\max _{a_{1} \in S_{i j k}} \sigma_{\max }\left(J\left(a_{0}\right)\right)}
$$

Where, $\omega_{i j k}$ represents the weight of the subspace $S_{i j k}, \sigma_{\min }$ and $\sigma_{\max }$ represent the minimum and maximum singular values of the Jacobian matrix respectively, $a_{0}$ and $a_{1}$ represent the positions of endeffector of minimum and maximum singular values of Jacobian matrix respectively, $\gamma_{i j k}$ represents the ratio of the accessible space of the terminal device to the operating space in the subspace $S_{i j k}$. The isotropic function GII $_{i j k}$ of the subspace $S_{i j k}$ is expressed as follows.

$$
G I I_{i j k}=\gamma_{i j k} \frac{\min _{a_{0} \in S_{i j k}} \sigma_{\min }\left(J\left(a_{0}\right)\right)}{\max _{a_{1} \in S_{i j k}} \sigma_{\max }\left(J\left(a_{1}\right)\right)}
$$

Second, multi-arm cooperation efficiency optimization function

Currently, surgical robots usually require coordinated operation of multiple arms holding the laparoscope and arms holding the robot. The ability of all the arm holding the robot to reach the designated position at the same time is called the multi-arm accessibility, and the designated position is within the scope of laparoscopic field of view at the end of the arm holding the laparoscope. The multi-arm collaboration capability function can be established to reflect the collaboration capability indexes of multi-arm accessibility and visibility of surgical robots. The larger the collaboration capability index is, the stronger the multi-arm coordination capability of the surgical robot is, which can be defined as the ratio of the volume of 
the cooperative space to the volume of the surgical workspace. The function expression is as follows:

$$
C C I=\sum_{i=0}^{A} \sum_{j=0}^{B} \sum_{k=0}^{C} \omega_{i j k} \frac{V_{m} \cap V_{w 1} \cap V_{w 2}}{V_{S_{i j k}}}
$$

Where, $V_{m}$ represents the reachable workspace of the arm holding the laparoscope in the subspace $S_{i j k}$, $V_{w 1}$ and $V_{w 2}$ respectively represent the reachable workspace of the two arms holding the robot in the subspace $S_{i j k}$, and $V_{S_{i j k}}$ represents the volume of the subspace $S_{i j k}$. The ratio of volume of cooperative space to volume of subspacein the subspace Sijk can be expressed as follows.

$$
C C I_{i j k}=\frac{V_{m} \cap V_{w 1} \cap V_{w 2}}{V_{S_{i j k}}}
$$

Third, multi-arm distance efficiency optimization function

After ensuring the isotropic performance of single arm and the collaboration performance of multi-arm of surgical robot, the collision between multiple mechanical arms should also be avoided. With the maximum distance method, the multi-arm distance function $(M D I)$ is constructed to represent the distance between multiple mechanical arms of the surgical robot. When constructing the distance function, joint 4 and the midpoint of the end rod of the mechanical arm are selected as the control point of wall hitting.

At this point, $M D I$ can be defined as the minimum distance between the selected control points in the cooperative space, as shown in Equation 9.

$$
M D I=\min _{a_{0} \in S_{c}}\left(\sum_{i=1}^{k} \sum_{j=1}^{k}\left|P_{a_{0}}\left(w_{1}, i\right)-P_{a_{0}}(m, j)\right|^{2}+\sum_{i=1}^{k} \sum_{j=1}^{k}\left|P_{a_{0}}(m, i)-P_{a_{0}}\left(w_{2}, j\right)\right|^{2}\right)
$$

Where, $k$ represents the number of wall-hitting control points, $S_{c}$ represents the collaborative space, and $P(l, i)$ represents the coordinate position of the ith wall-hitting control point of the corresponding mechanical arm. The distance between two wallhitting control points can be expressed as follows.

$$
\left|P\left(w_{1}, i\right)-P(m, j)\right|=\sqrt{\left(P_{x}\left(w_{1}, i\right)-P_{x}(m, j)\right)^{2}+\left(P_{y}\left(w_{1}, i\right)-P_{y}(m, j)\right)^{2}+\left(P_{z}\left(w_{1}, i\right)-P_{z}(m, j)\right)^{2}}
$$

\subsection{Particle swarm optimization - Gaussian process (PSO-GP)}

In the real environment, the calculation amount of the optimization objective function is too large. When adopting particle swarm optimization (PSO) to optimize, it is necessary to reduce the number of iterations and obtain the optimization result with good effect. The Gaussian process is combined with particle swarm optimization algorithm. In each iteration of the particle swarm optimization

$$
\begin{aligned}
& D_{1[k]}=\left\{\begin{array}{l}
x=\left[P_{w 1[i]}, \theta_{w 1[i]}\right] \\
y=\left[G I I_{w 1[i]}\right]
\end{array}\right\}_{i=1,2, \ldots, k} \\
& D_{2[k]}=\left\{\begin{array}{l}
x=\left[P_{w 2[i]}, \theta_{w 2[i]}\right] \\
y=\left[G I_{w 2[i]}\right]
\end{array}\right\}_{i=1,2, \ldots, k} \\
& \left.D_{3[k]}=\left\{\begin{array}{l}
x=\left[P_{m[i]}, \theta_{m[i]}\right] \\
y=\left[G I I_{m[i]}\right]
\end{array}\right\}_{i=1,2, \ldots, k}, \theta_{w 2[k]}, P_{m[i]}, \theta_{m[i]}\right] \\
& D_{4[k]}=\left\{\begin{array}{l}
x=\left[P_{w 1[i]}, \theta_{w 1[i]}, P_{w 2[k]}, \theta_{w i, 2, \ldots, k}\right. \\
y=\left[C C I_{[i]}, M D I_{[i]}\right]
\end{array}\right.
\end{aligned}
$$

algorithm, the gaussian process is adopted to estimate the global optimal value, and then compared with the real adaptive value and the global particle swarm history value.

The optimal value of the two is taken into the iterative equation, and the optimal position of the arm holding the robot is found through continuous iteration.

The training set of the Gaussian process is obtained from the real adaptive value obtained in the completed iterative steps, as shown in Equation 11.
$P_{4}$ and $\theta_{4}$ are the position and rotation angle of joint 4 , respectively.
Where, $x$ is the input of the training set and $y$ is the output of the training set.

$$
\begin{aligned}
& P_{4}=\left[P_{w 1}, P_{w 2}, P_{m}\right]^{T} \\
& \theta_{4}=\left[\theta_{w 1}, \theta_{w 2}, \theta_{m}\right]^{T}
\end{aligned}
$$


Solving $P_{4}$ and $\theta_{4}$ can be regarded as an optimization problem with constraints.

$$
\left(P_{4}^{*}, \theta_{4}^{*}\right)=\underset{P_{4}, \theta_{4}}{\arg \max } L\left(P_{4}, \theta_{4}\right)
$$

The condition that satisfies the constraints is as follows.

$$
\begin{aligned}
& v_{i \text { min }} \leq v_{i} \leq v_{i \text { max }} \\
& P_{4 \text { min }} \leq P_{4} \leq P_{4 \text { max }} \\
& \theta_{4 \text { min }} \leq \theta_{4} \leq \theta_{4 \text { max }}
\end{aligned}
$$

According to the actual structure of the surgical robot, the position and motion range and velocity of each particle are shown in Table 2.

Table 2. Constraint conditions of particle swarm model (unit: $\mathrm{mm}$, rad)

\begin{tabular}{|c|c|c|c|c|c|c|}
\hline & $p_{x}$ & $p_{y}$ & $\theta$ & $V_{\max }\left(p_{x}\right)$ & $V_{\max }\left(p_{y}\right)$ & $V_{\max }(\theta)$ \\
\hline Arm holding the robot (left) & {$[-700,-500]$} & {$[-550,0]$} & {$[0,90]$} & 45 & 35 & 6 \\
\hline Arm holding the robot (right) & {$[-700,-500]$} & {$[-800,500]$} & {$[-30,30]$} & 45 & 35 & 6 \\
\hline Arm holding the robot (middle) & {$[-800,-500]$} & {$[-500,500]$} & {$[-90,0]$} & 45 & 35 & 6 \\
\hline
\end{tabular}

Considering the combined effects of the above three kinds of performance, the comprehensive objective optimization function $L\left(P_{4}, \theta_{4}\right)$ is defined as the combined function of GII, CCI, and MDI, as shown in Equation (16).

$$
L\left(P_{4}, \theta_{4}\right)=\omega_{1}\left(G I I_{w 1}+G I I_{w 2}+G I I_{m}\right)+\omega_{2} C C I+\omega_{3} M D I
$$

Among them, $\omega_{1}, \omega_{2}$, and $\omega_{3}$ are weights, which make the values of $\omega_{1}\left(G I I_{w 1}+G I_{w 2}+G I I_{m}\right), \omega_{2} C C I$, and $\omega_{3} M D I$ the same.

Combining particle swarm optimization [14] with gaussian process [15], the comprehensive optimization algorithm is as follows:

\section{PSO-GP algorithm}

1. Input: $P_{w 1}^{0} ; \theta_{w 1}^{0} ; P_{w 2}^{0} ; \theta_{w 2}^{0} ; P_{m}^{0} ; \theta_{m}^{0}$

2.Output: optimal poses $P_{w 1}^{*} ; \theta_{w 1}^{*} ; P_{w 2}^{*} ; \theta_{w 2}^{*} ; P_{m}^{*} ; \theta_{m}^{*}$

3. initialize $P^{0}, \theta^{0}$; calculate $G I I, C C I, L$

4. obtain $B_{g}^{0}, B_{i}^{0}, L_{p}^{0}$

\section{5. for iteration $(k)=1$ to $m$ do}

6. $\left(x_{r g}^{k-1}, L_{g}^{k-1}\right)=\operatorname{learnGP}\left(P^{k-1}, \theta^{k-1}\right)$

7. calculate actual $L_{g}^{(k-1) *}$ with eq. (5), (7), (9), (16)

8. if $L_{g}^{(k-1) *}>L_{p}^{(k-1)}$ then

9. $B_{g}^{(k-1) *}=x_{r g}^{k-1}$

10. else

11. $B_{g}^{(k-1) *}=B_{g}^{(k-1)}$

12. end

13. $B_{g}^{(k-1)}=B_{g}^{(k-1) *}$

14. for $\operatorname{size}(i)=1$ to $n$ do

15. update $v_{i}^{k}, x_{i}^{k}$ with

$v_{i}^{k+1}=\omega v_{i}^{k}+c_{1} \cdot \operatorname{Rand}_{1}\left(B_{i}^{k}-x_{i}^{k}\right)+c_{2} \cdot \operatorname{Rand}_{2}\left(B_{g}^{k}-x_{i}^{k}\right)$

$x_{i}^{k+1}=x_{i}^{k}+r v_{i}^{k+1}$
16. calculate GII, CCI, L with eq. (5), (7)

17. end

18. obtain $L_{p}^{k}$ with eq. (14)

19. end

As shown in steps 6 and 7, firstly, the motion space of the joint in Table 2 is discretized, and each discrete point can be represented by a group $\left(P_{4}, \theta_{4}\right)$. After $\mathrm{k}$ times of particle swarm optimization, the global optimal value $L_{g}^{k}$ and the corresponding optimal position $x_{r g}^{k}$ are estimated through the training set of Equation 11, and then the corresponding real adaptive value $L_{g}^{k *}$ is calculated.

As shown in step 8-13, $L_{g}^{k *}$ and the historical global optimal value $L_{p}^{k}$ of particle swarm optimization algorithm is compared. The largest value is taken as the historical global optimal value, and the corresponding particle position would be regarded as the updated global optimal position $B_{g}^{k *}$.

As shown in steps 14-18, $B_{g}^{k *}$ would be treated as $B_{g}^{k}$, the comprehensive optimal objective function value $L_{p}^{k}$ is calculated, and the next iteration optimization is done in the same way.

\subsection{Construction of virtual simulation system for multi-arm surgical robot}

The process of establishing a virtual simulation system is as follows.

First, the surgical robot is geometrically modeled by Solid Works software, the kinematic coordinate system is established, the pose of each rod piece is calculated, and the file is saved as .step format and exported. 
Second, the .step file is opened in the 3Ds MAX software and converted to the .3DS format.

Third, the .3DS file is poured into CHAI3D, and the kinematics program is written to control the robot movement through the Omega.7 main operator.

Among them, CHAI3D is a software based on $\mathrm{C}++$. The process adopting CHAI3D is as follows: the cWorld class is called to create a virtual environment; the cCamera class is called to create a virtual camera and provide a perspective of the virtual environment; the cLight class is called to establish lighting; the cMesh class is called to load the robot model into the virtual environment and determine the pose of each rod piece according to the kinematics.

According to the above optimization methods, the optimal results of preoperative positioning are obtained. When the optimization results are input in turn, the passive arm of the virtual robot will move to the corresponding pose based on the kinematic model, so as to obtain the position of the telecentric point. The system shows the optimized arm position and incision position to help doctors preview the preoperative configuration of the surgical machine.

The pre-operative positioning of the virtual robot and the preview process of the surgery are shown in Figure 5. According to the optimization results, the passive joint is rotated to obtain the position of incision operation.

The active joint is controlled by the main operator, the position of the active joint of the virtual surgical robot is determined, and a surgical preview is performed. Finally, the status of the robot is revealed according to the established virtual simulation system.

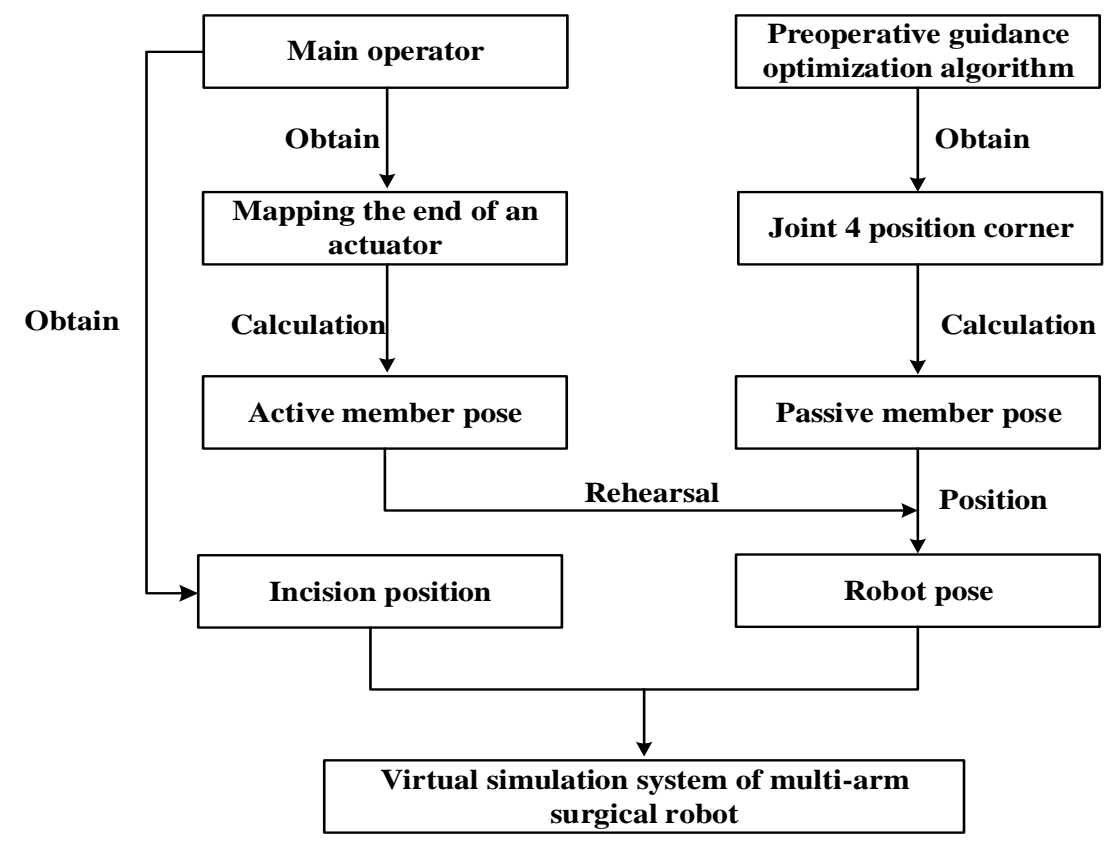

Figure 4: Preoperative positioning and surgical rehearsal flow of a virtual robot

\section{Results}

\subsection{Experimental facility}

According to the above Figure 3 , the surgical work space is divided into $3 \times 3 \times 2$ subspaces.

To ensure that the endoscopic arm has enough field of vision, the upper space of the surgical space (layer $q_{1}$ ) is taken as the reserved space, and the weight of each subspace is set to 0 .
Four groups of experiments on weight distribution of different subspaces are carried out. The weight distribution is shown in Table 3.

The fourth group of experiments is a comparison experiment that does not consider the internal differences in the workspace, and 40 iteration experiments are performed according to this weight distribution.

Table 3. Distribution of weights in the subspace

\begin{tabular}{|c|c|c|c|c|c|c|c|c|c|c|}
\hline \multirow{2}{*}{$\begin{array}{l}\text { Experimental } \\
\text { group }\end{array}$} & \multirow{2}{*}{$\begin{array}{c}q_{1} \\
\text { layer }\end{array}$} & \multicolumn{9}{|c|}{ The distribution of weights in subspaces } \\
\hline & & $S_{020}$ & $S_{120}$ & $S_{220}$ & $S_{010}$ & $S_{110}$ & $S_{210}$ & $S_{000}$ & $S_{100}$ & $S_{200}$ \\
\hline 1 & 0 & 0.20 & 0.21 & 0.05 & 0.20 & 0.24 & 0.05 & 0.00 & 0.05 & 0.00 \\
\hline 2 & 0 & 0.05 & 0.23 & 0.22 & 0.05 & 0.21 & 0.19 & 0.00 & 0.05 & 0.00 \\
\hline 3 & 0 & 0.06 & 0.23 & 0.15 & 0.05 & 0.41 & 0.05 & 0.00 & 0.05 & 0.00 \\
\hline 4 & \multicolumn{10}{|c|}{ No weight } \\
\hline
\end{tabular}




\subsection{Iterative experiment}

The iterative experiment is performed with the weight distribution of the first group. Table 4 and Figure 5 show the position of the robot arm and the incision under different iterations, that is, the position and angle of joint 4 of surgical robot, the value of global isotropic optimization function and multi-arm coordination function. As can be observed from the data in the table, with the increase of iteration times, the values of $G I I$ and $C C I$ functions tend to increase, that is, the performance of the multi-arm surgical robot gradually improves, indicating the effectiveness of PSO-GP algorithm.

Table 4. Position and rotation angle of joint 4 (unit: $\mathrm{mm}$, rad)

\begin{tabular}{|l|c|c|c|c|c|c|c|c|c|}
\hline \multirow{2}{*}{ Number of iterations } & \multicolumn{3}{|c|}{10} & \multicolumn{3}{c|}{20} & \multicolumn{3}{c|}{40} \\
\cline { 2 - 10 } & $p_{x}$ & $p_{y}$ & $\theta_{1}$ & $p_{x}$ & $p_{y}$ & $\theta_{1}$ & $p_{x}$ & $p_{y}$ & $\theta_{1}$ \\
\hline Arm holding the robot (left) & -681.9 & -436.7 & -0.9 & -548.2 & -503.1 & -1.0 & -507.4 & -520.6 & -1.0 \\
\hline Arm holding the robot (right) & -643.6 & 427.6 & -1.8 & -628.1 & 425.8 & -2.0 & -593.2 & 394.5 & -1.9 \\
\hline $\begin{array}{l}\text { Arm holding the robot } \\
\text { (middle) }\end{array}$ & -627.4 & 284.5 & -1.1 & -615.7 & -153.9 & -1.5 & -584.3 & -106.7 & -1.5 \\
\hline
\end{tabular}
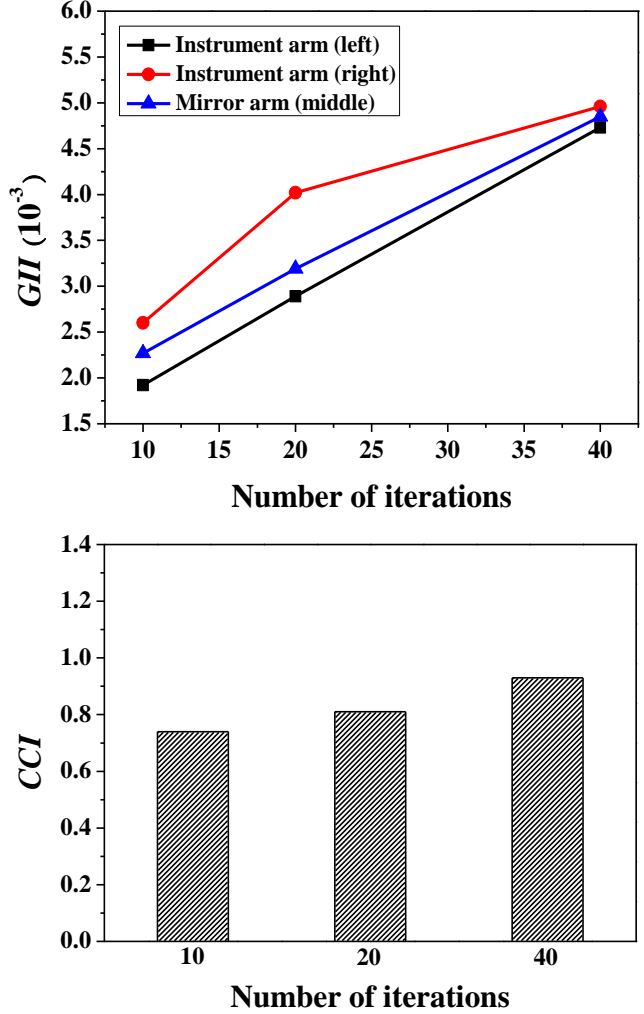

Figure 5: Objective function values for different iterations

Figure 6 shows the distribution of the cooperative space in the subspace when the number of iterations is 40 , and the green area represents the cooperative space.

As can be observed from the figure, the cooperative space in the subspaces $S_{010}, S_{110}, S_{020}$, and $S_{120}$ is relatively large, and the weights assigned before the simulation are also relatively high $(0.20$, $0.24,0.20$, and 0.21 , respectively), which can be used as the hot area of surgery.
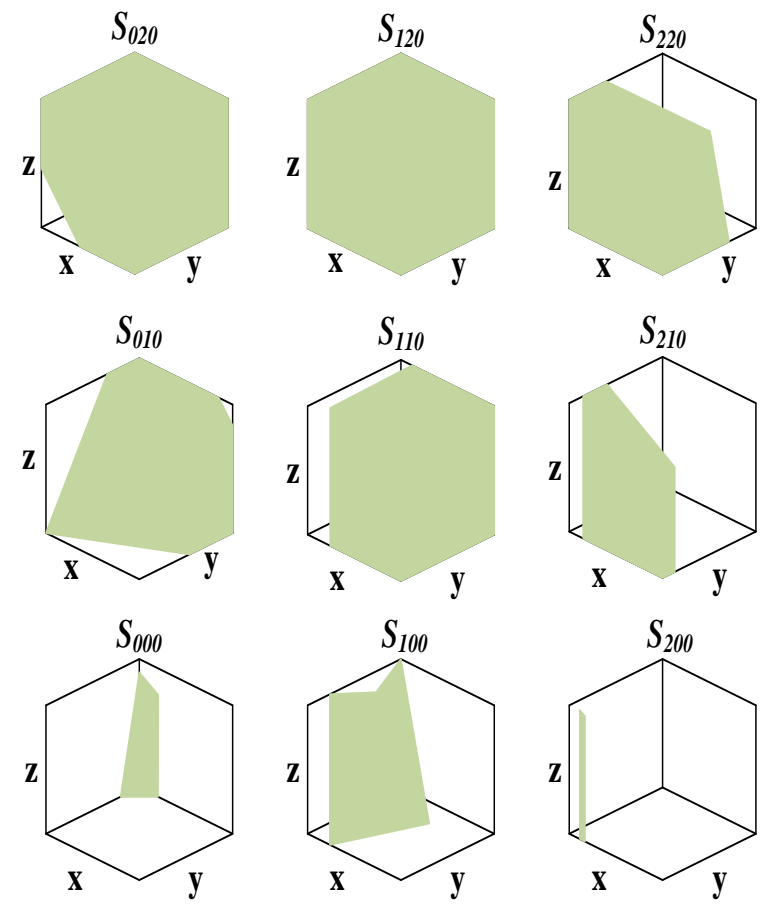

Figure 6: Distribution of co-operative spaces in subspaces

Under the same conditions, PSO-GP algorithm and PSO algorithm are respectively iterated for 40 times to compare and study the optimization speed and accuracy of PSO-GP algorithm, as shown in Figure 7.

As can be observed from the figure, the overall $L$ value of the comprehensive objective optimization function obtained by PSO-GP algorithm is larger than that obtained by PSO algorithm, indicating that the accuracy of PSO-GP algorithm is higher and the number of iterations required is less. 


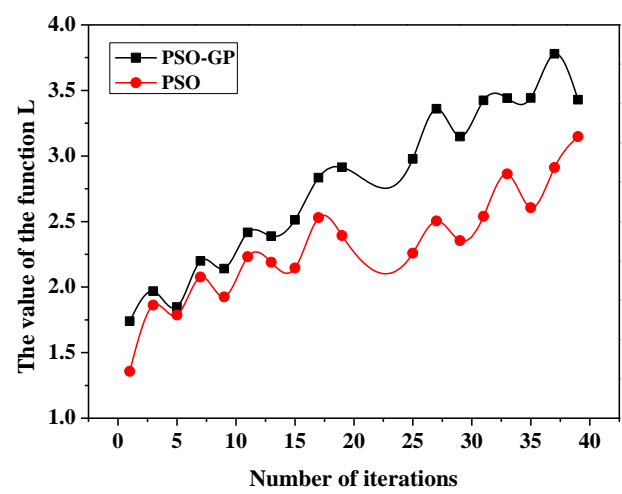

Figure 7: Online optimization experiment results

At the same time, to study the universality of the proposed PSO-GP algorithm, four groups of experiments with different weight distribution are conducted. Figure 8 shows the $C C I_{i j k}$ and weights distribution in 9 subspaces, the subspaces $1,2,3 \ldots$

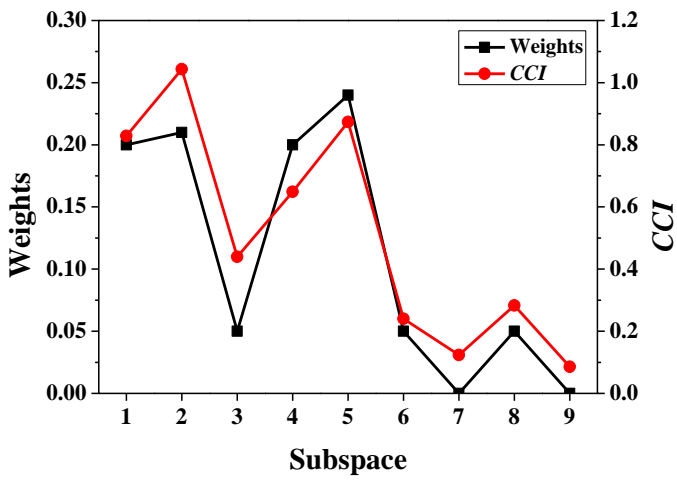

(a)

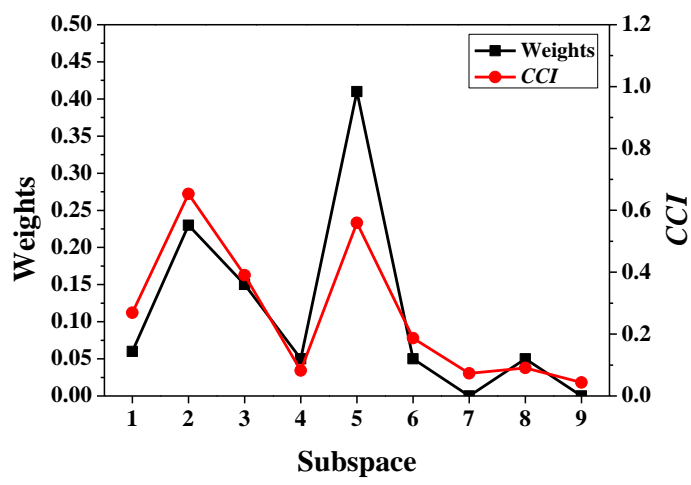

(c) correspond to the position subspaces $S_{020}, S_{120}, S_{220}$, etc. in that order.

As can be observed from the figure, the $C C I_{i j k}$ distribution of the subspace is closely related to its weight distribution, and the higher the weight, the larger the $C C I_{i j k}$ value obtained. Taking group 1 as an example, according to the weight distribution of the subspace in Table 3, it can be concluded that $S_{020}$, $S_{120}, S_{010}$, and $S_{110}$ have higher weights, and the corresponding $C C I$ values are also larger.

The results of the second group and the third group are the same.

From the fourth group of experiments, it can be concluded that without considering the weight of the subspace, the $C C I_{i j k}$ values in the central part of the surgical workspace are all higher, while the values in the edge part are lower, which can't ensure that the multi-arm surgical robot has a higher efficiency in the hot spot area.

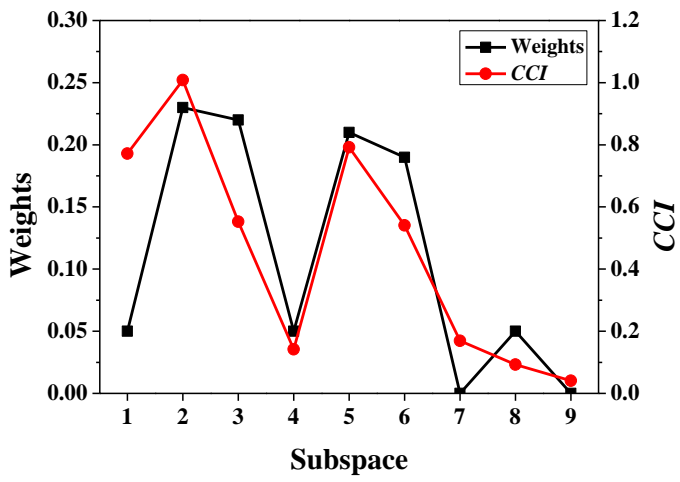

(b)

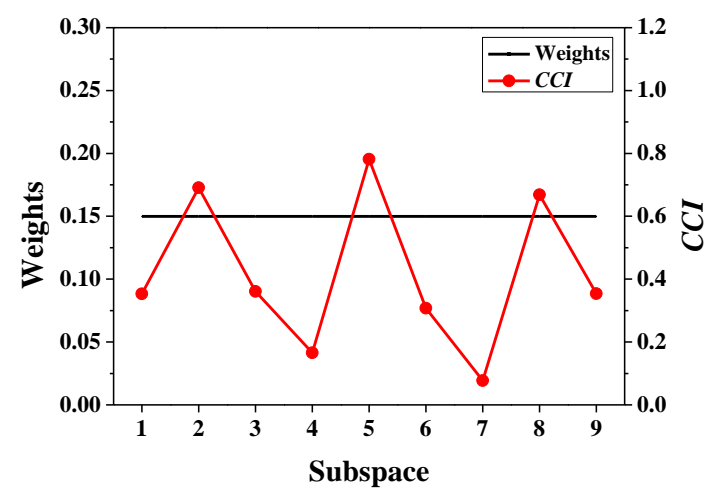

(d)

Figure 8: CCI ${ }_{i j k}$ and weight distribution in the subspace

\section{Discussion}

In this study, a comprehensive optimization algorithm (PSO-GP) combining particle swarm optimization with gaussian process is proposed based on the performance index of the multi-arm surgical robot. The effectiveness and rationality of the algorithm are verified in the virtual simulation system, so as to optimize the performance of multiarm surgical robot for clinical treatment.
The results show that PSO-GP algorithm is effective, feasible, and reasonable, with high precision and in line with the expected results. During surgery, the ends of the arm holding the laparoscope and the ends of the two arms holding the robot need to be concentrated into a single space for operation, and the end of the arms holding the robot must be placed within the field of vision.

The end of the robotic arm will not be concentrated in one place, and the position change 
needs to be performed according to the surgical hotspot. Therefore, it is necessary to constantly adjust the position of the robot arm. In this study, during the iteration process of the particle swarm optimization algorithm, the gaussian process is used to predict the global optimal value, and the global optimal value is compared with the real adaptive value and the historical global optimal value of particle swarm optimization, so as to find the optimal preoperative positioning of the multi-arm surgical robot iteratively.

The results show that the PSO-GP algorithm is efficient, has high optimization accuracy, and few iterations, so the robot has high efficiency in the hot spot area of surgery.

Accordingly, $\mathrm{Wu}$ et al. [16] combined particle swarm optimization algorithm with gaussian process, which could improve the convergence speed and have better performance and stability.

\section{Conclusion}

To optimize the performance of the multi-arm surgical robot and realize it in the virtual simulation system to achieve better surgical effect, a comprehensive optimization algorithm combining particle swarm optimization algorithm with gaussian process is proposed in this research according to the performance index of the multi-arm surgical robot.

Based on this, a virtual simulation system is constructed and the proposed algorithm is iterated in the virtual simulation system to verify the effectiveness and rationality. With the increase of the number of iterations, the values of global isotropic optimization function and the multi-arm cooperation function gradually increase, and the efficiency of the multi-arm surgical robot also increases, so the proposed algorithm is effective and feasible.

The total value of the integrated objective optimization function obtained by the proposed algorithm is larger than that of the particle swarm optimization algorithm, with higher precision and fewer iterations. Without considering the weight of the subspace, it is impossible to guarantee that the multi-arm surgical robot has a high efficiency in the hot spot area.

The proposed algorithm can optimize the performance of the multi-arm surgical robot, has good feasibility and precision, and achieves good surgical effect.

However, the virtual simulation system can't provide the real surgical environment, which can be separated from the real surgical robot, thus affecting the effect. In the follow-up study, the virtual and real fusion technology will be considered to assist doctors in the preoperative positioning of multi-arm surgery robots.

\section{References}

[1] André H., Kelly R. E., Ferro M. M, et al. (2017) Life-threatening complications and mortality of minimally invasive pectus surgery. Journal of Pediatric Surgery, 53(4), 728-732.

[2] Yang J., Yu L., Wang L, et al. (2017) Preoperative Planning of a Celiac Minimally Invasive Surgery Robot Based on Feature Parameters and Double Collaboration Space. Robot, 39(2), 230-238.

[3] Zeng B., MengF., Ding H, et al. (2017) A surgical robot with augmented reality visualization for stereo electroencephalography electrode implantation. International Journal of Computer Assisted Radiology and Surgery, 12(3), 1-14.

[4] Toru S., Hideo Y., Hiroki M, et al. (2017) Accessibility to surgical robot technology and prostate-cancer patient behavior for prostatectomy. Japanese Journal of Clinical Oncology, 47(7), 1-5.

[5] Wang T., Pan B., Fu Y, et al. (2018) Minimally invasive surgical robot based on EtherCAT bus and TwinCAT motion controller. Journal of Huazhong University of Science and Technology (Natural Science Edition), 46(12), 1-7.

[6] Li J. H., Shen J. C., Su H, et al. (2018) Method for Latency Measurement of Visual Feedback-Based Master-Slave Minimally Invasive Surgical Robot. Transactions of Tianjin University, 24(4), 375386.

[7] Shunsuke T., Yuji N., Hiroki O, et al. (2017) Surgical outcomes of robot-assisted rectal cancer surgery using the da Vinci Surgical System: A multi-center pilot Phase II study. Japanese Journal of Clinical Oncology, 47(12), 1-6.

[8] Aldair A. A., Rashid A. T., Rashid M. T, et al. (2019) Adaptive Fuzzy Control Applied to Seven-Link Biped Robot Using Ant Colony Optimization Algorithm. Iranian Journal of Science and Technology-Transactions of Electrical Engineering, 43(4), 797-811.

[9] Ros-Freixedes L., Gao A., Liu N, et al. (2019) Design optimization of a contact-aided continuum robot for endobronchial interventions based on anatomical constraints. International Journal of Computer Assisted Radiology and Surgery, 14(3), 1137-1146.

[10] Watanabe K., Kanno T., Ito K, et al. (2017) Single-Master Dual-Slave Surgical Robot With Automated Relay of Suture Needle. IEEE Transactions on Industrial Electronics, 65, 63436351. 
[11] Zhang F. H., Qu J. D., Liu H, et al. (2019) A MultiPriority Control of Asymmetric Coordination for Redundant Dual-Arm Robot. International Journal of Humanoid Robotics, 16(1), 1-25.

[12] Wang Z. Y., Zi B., Ding H. F. (2018) Hybrid grey prediction model-based autotracking algorithm for the laparoscopic visual window of surgical robot. Mechanism \& Machine Theory, 123,107123.

[13] Zhang L., Cao T., Liu D. (2017) Optimization of end-tool parameters based on robot hand-eye calibration. Journal of biomedical engineering, 34(2), 271-277.
[14] Mohammad R. B., Zbigniew M. (2017) Particle Swarm Optimization for Single Objective Continuous Space Problems: A Review. Evolutionary Computation, 25(1), 1-54.

[15] Richardson, Robert R., Osborne, Michael A., Howey, David A. (2017) Gaussian process regression for forecasting battery state of health. Journal of Power Sources, 357, 209-219.

[16] Wu R. X., Sun H., Zhu D. G, et al. (2016) Particle Swarm Optimization Algorithm Based on Optimal Particle Guidance and Gauss Perturbance. Journal of Chinese Computer Systems, 37(1), 146.151. 